\title{
Vibration Measurement of a Screening Machine
}

\author{
L. RÓNAI ${ }^{1}$, J. LÉNÁRT ${ }^{2}$, T. SZABÓ3 \\ 1University of Miskolc, Faculty of Mechanical Engineering and Informatics, Robert Bosch Department of \\ Mechatronics, ronai.laszlo@uni-miskolc.hu \\ 2 University of Miskolc, Faculty of Mechanical Engineering and Informatics, Robert Bosch Department of \\ Mechatronics, lenart.jozsef@uni-miskolc.hu \\ ${ }^{3}$ University of Miskolc, Faculty of Mechanical Engineering and Informatics, Robert Bosch Department of \\ Mechatronics, szabo.tamas@uni-miskolc.hu
}

Abstract. In this paper we use linear vibration approach to make a model of a horizontal vibration screen, which can be applied at gravel pit in order to perform separation operation of the wet gravel particles. The model can produce the natural frequencies of the system, which are useful in the adjustment of the angular velocity of the vibration exciter. A laser triangulation unit is well applicable to measure peak-to-peak amplitude of the working vibration screen. Comparison has been performed between the results of the simulation and the measurements.

Keywords: screening machine, linear vibration approach, laser triangulation

\section{Introduction}

Nowadays automation is widely used to improve productivity also in mining machinery industry. Gravel pits ensure the opportunity of the extraction of gravel, where gravels are separated based on its weight by a gravel separator/screening machine, which has a vibrating mechanism in order to perform a separation process.

Screen machines play a major role in the mining processing techniques, i.e., the efficient separation of mineral particles into various sizes. These type of systems have continuously been improved over the recent years [1]. Reconfigurable vibration screens [1], [2] are effective, which can be configured in order to serve the current mineral demand. Some papers deal with the design methodology and optimization of vibration screens [2], [3]. Modal analysis is well applicable to examine the dynamic characteristic of a vibrating screen machine in its design process [3]. Usually an automated horizontal screen ensures the vibrating mechanism with eccentric weights.

In this paper a Weir Trio® TTH6163 screening machine [4], [5] is examined. The task was raised by a Hungarian gravel mining company to do an analysis of how its screening machine works. This machine has three eccentric timed vibration exciter shafts with oval counterweights, which are applicable to provide an oval stroke with adjustable amplitude [4]. It has three different decks with steel screen panels in order to isolate different sized particles at the same time. The unit can ensure wet or dry applications and easy stroke, speed adjustments [4]. 
This paper is organized as follows: Section 1 deals with the modelling and simulation of the screening machine. Linear vibration approach is used to produce the differential equation of the system. Modal analysis is applied to solve the eigenvalue-eigenvector problem of the unit [6]. Furthermore, measurements are performed in order to validate the model. The concluding remarks are given in the last Section.

\section{Model of the screening machine}

The vibration of the Weir Trio® TTH6163 [4], [5] screening machine (see Figure 1) is modelled with a $6 \mathrm{DoF}$ 3D linear system. Screening machine can be considered as a rigid body supported by eight rubber pads (see Figure 3.), which have three directional stiffness properties. The system is excited with three interconnected eccentric weights. Modal analysis is applied to solve numerically the equations of motion, taking into consideration of uniform modal damping.

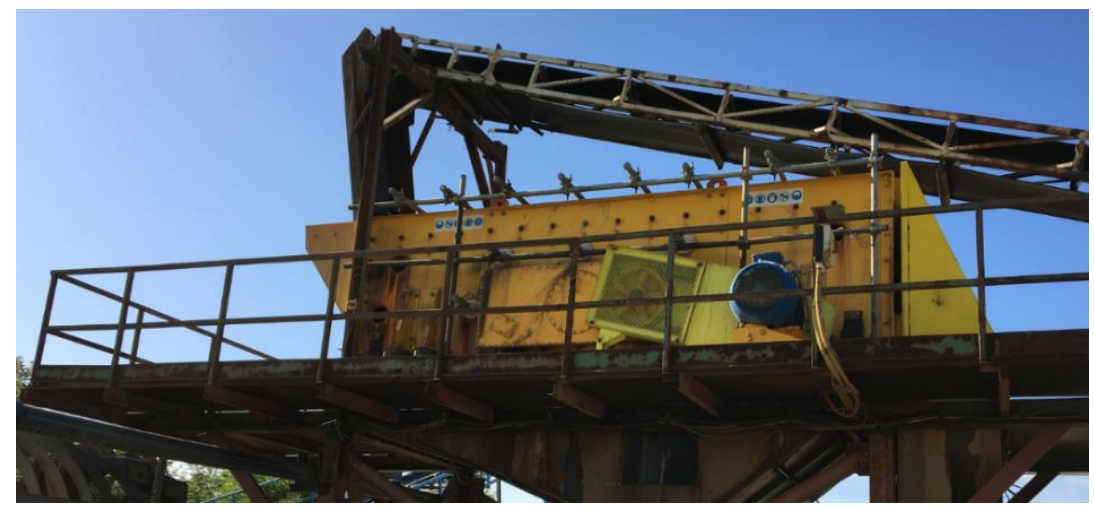

Figure 1. The screening machine

The structure of the system is shown in Figure 2. The system has 6 degree of freedoms, i.e., displacements $\vec{u}_{d}=u_{s} \cdot \vec{\imath}+v_{s} \cdot \vec{\jmath}+w_{s} \cdot \vec{k}$ and small angular rotations $\vec{\varphi}=\varphi_{x} \cdot \vec{\imath}+\varphi_{y} \cdot \vec{\jmath}+\varphi_{z} \cdot \vec{k}$ in direction of the coordinate axes. The supporting rubber pads can be considered by 12 springs given in coordinate directions at the four lower corners.

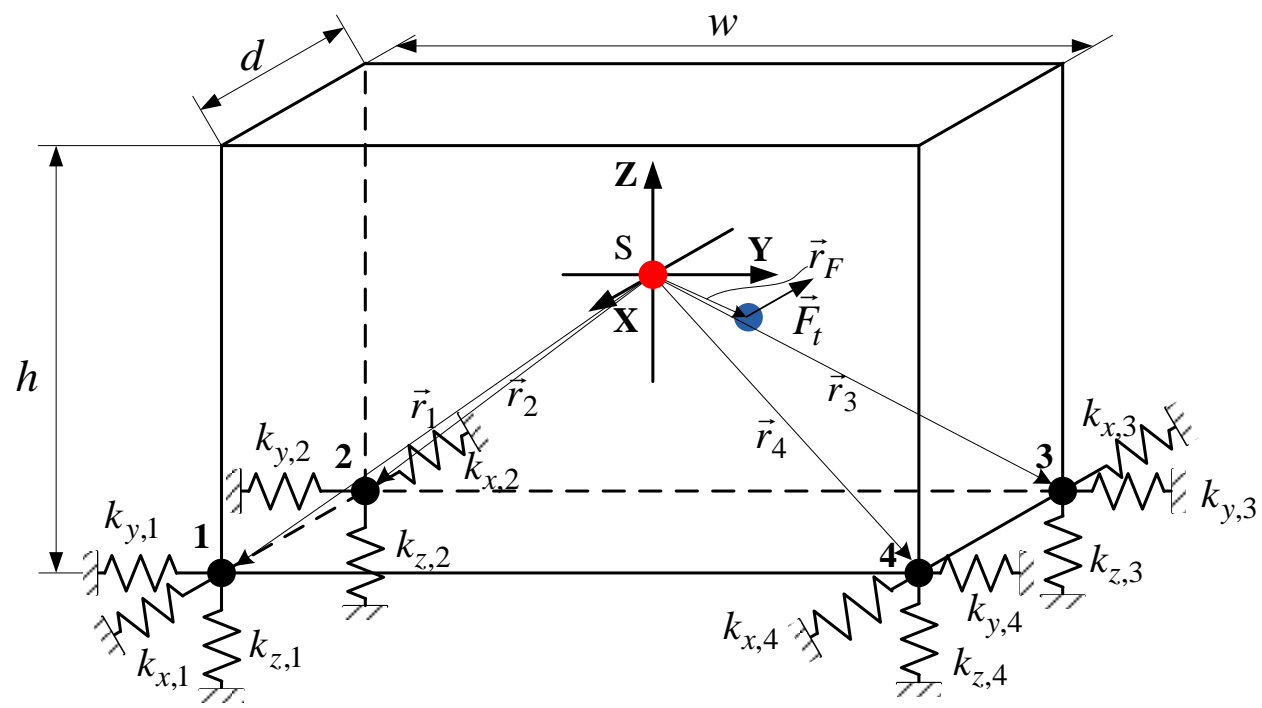

Figure 2. The model of the screening machine 
The system is considered as a rigid cuboid, which has height, width and depth. The geometric and mass specifications of the model are given in Table 1.

\begin{tabular}{|c|c|}
\hline Name & Value \\
\hline Height (h) & $1,974 \mathrm{~m}$ \\
\hline Width (d) & $5,604 \mathrm{~m}$ \\
\hline Depth (w) & $3,364 \mathrm{~m}$ \\
\hline Mass (m) & $8577 \mathrm{~kg}$ \\
\hline
\end{tabular}

Table 1. Parameters of the model

The tensor of moment of inertia referring to the centre of gravity, is denoted by $\mathbf{J}_{\mathbf{S}}$. The entries of the moments of inertia tensor are determined by the following assumptions [7]:

$$
\begin{aligned}
& J_{s, z}=\frac{1}{12} m\left(w^{2}+d^{2}\right)=30535 \mathrm{kgm}^{2}, \\
& J_{s, y}=\frac{1}{12} m\left(d^{2}+h^{2}\right)=10874 \mathrm{kgm}^{2}, \\
& J_{s, x}=\frac{1}{12} m\left(w^{2}+h^{2}\right)=25232 \mathrm{kgm}^{2} .
\end{aligned}
$$

Eight pieces of SRX 100-160 [8] rubber pads in pairs support the body at the vicinity of the lower corners. The resultant stiffness of a pair of pads is uniformly applied in three directions:

$$
k_{x, i}=k_{y, i}=k_{z, i}=7.92 \cdot 10^{5} \frac{\mathrm{N}}{\mathrm{m}}, \quad i=1,2,3,4 .
$$

The position vectors of the four corners are denoted by $\vec{r}_{i},(i=1,2,3,4)$, the position vector $\vec{r}_{F}$ of the point of application of the force due to rotating eccentric, which is a harmonic function of time.

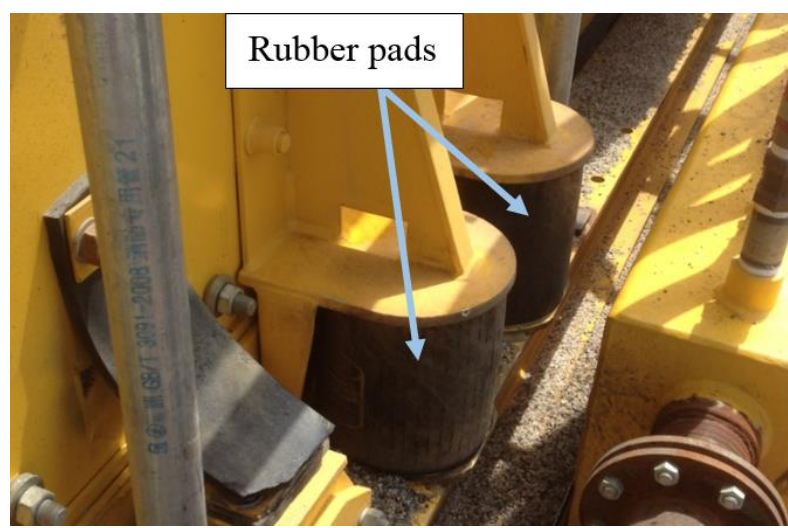

Figure 3. The rubber springs located on the base of the system

The point of application of the force and the position vectors of the corners can be written [9]

$$
\mathbf{u}_{\mathbf{F}}=\mathbf{u}_{\mathbf{d}}+\mathbf{R}_{\mathbf{F}} \cdot \boldsymbol{\varphi} ; \quad \mathbf{u}_{\mathbf{i}}=\mathbf{u}_{\mathbf{d}}+\mathbf{R}_{\mathbf{i}} \cdot \boldsymbol{\varphi}, \quad i=(1,2,3,4),
$$

where the matrices of $\mathbf{R}_{\mathbf{F}}$ and $\mathbf{R}_{\mathbf{i}}$

$$
\mathbf{R}_{\mathbf{F}}=\left[\begin{array}{ccc}
0 & z_{F} & -y_{F} \\
-z_{F} & 0 & x_{F} \\
y_{F} & -x_{F} & 0
\end{array}\right] ; \quad \mathbf{R}_{\mathbf{i}}=\left[\begin{array}{ccc}
0 & z_{i} & -y_{i} \\
-z_{i} & 0 & x_{i} \\
y_{i} & -x_{i} & 0
\end{array}\right], \quad i=(1,2,3,4) .
$$

The strain energy of the springs [6]: 


$$
U=\sum_{i=1}^{4}\left(\frac{u_{i}^{2}}{2} k_{x, i}+\frac{v_{i}^{2}}{2} k_{y, i}+\frac{w_{i}^{2}}{2} k_{z, i}\right),
$$

which can be written in matrix form

$$
U=\sum_{i=1}^{4}\left(\frac{1}{2} \mathbf{u}_{\mathbf{i}}^{\mathbf{T}} \mathbf{K}_{\mathbf{i}} \mathbf{u}_{\mathbf{i}}\right),
$$

where $\mathbf{K}_{\mathbf{i}}$ is the stiffness matrix at the $i^{\text {th }}$ corner. After some transformations the final form of the strain energy is written:

$$
U=\frac{1}{2}\left[\begin{array}{ll}
\mathbf{u}_{\mathbf{d}}^{\mathbf{T}} & \boldsymbol{\varphi}^{\mathrm{T}}
\end{array}\right] \mathbf{K}\left[\begin{array}{c}
\mathbf{u}_{\mathbf{d}} \\
\boldsymbol{\varphi}
\end{array}\right],
$$

where $\mathbf{K}$ is the global stiffness matrix.

The kinetic energy of the screening machine can be formulated as

$$
E=\frac{1}{2}\left(\dot{\mathbf{u}}_{\mathbf{d}}^{\mathbf{T}} \mathbf{m} \dot{\mathbf{u}}_{\mathbf{d}}+\dot{\boldsymbol{\varphi}}^{\mathrm{T}} \mathbf{J}_{\mathbf{S}} \dot{\boldsymbol{\varphi}}\right),
$$

m denotes the mass matrix.

Lagrange's equations of the second kind is applicable to produce the equation of the motion of the system:

$$
\frac{d}{d t}\left(\frac{\partial \mathcal{L}}{\partial \dot{\mathbf{q}}}\right)-\frac{\partial \mathcal{L}}{\partial \mathbf{q}}=\mathbf{Q}_{\mathbf{F}}
$$

where $\mathcal{L}=E-U$ is the Lagrange's function, $\mathbf{q}$ and $\dot{\mathbf{q}}$ are the generalized coordinates and velocities, respectively:

$$
\mathbf{q}=\left[\begin{array}{c}
\mathbf{u}_{\mathbf{d}} \\
\boldsymbol{\varphi}
\end{array}\right], \quad \dot{\mathbf{q}}=\left[\begin{array}{c}
\dot{\mathbf{u}}_{\mathbf{d}} \\
\dot{\varphi}
\end{array}\right] .
$$

Performing the derivations in (11) the equation the motion can be written in matrix form as:

$$
\mathbf{M} \ddot{\mathbf{q}}+\mathbf{K} \mathbf{q}=\mathbf{Q}_{\mathbf{F}},
$$

where $\mathbf{M}$ is the mass matrix of the system, $\mathbf{K}$ is the stiffness matrix, and $\mathbf{Q}_{\mathbf{F}}$ is the matrix of the generalized forces. The initial condition is $\mathbf{q}(0)=\mathbf{q}^{0}, \dot{\mathbf{q}}(0)=\dot{\mathbf{q}}^{0}$.

Modal analysis includes the solution of an eigenvalue problem [6]. Searching the solution with a harmonic time function

$$
\mathbf{q}=\mathbf{q}_{\mathbf{0}} \cos (\alpha t),
$$

where $\mathbf{q}_{\mathbf{0}}$ is the vector of amplitude and $\alpha$ is the natural frequency.

The eigenvalue problem is formulated as follows

$$
\left(\mathbf{K}-\alpha_{i}^{2} \mathbf{M}\right) \mathbf{q}_{\mathbf{0}, \mathbf{i}}=0, \quad(i=1,2, \ldots, 6),
$$

where $\mathbf{q}_{\mathbf{0}, \mathbf{i}}$ is the eigenvector. It is advisable to normalize the eigenvector [9]: 


$$
\mathbf{q}_{\mathbf{n}, \mathbf{i}}=\frac{\mathbf{q}_{\mathbf{0}, \mathbf{i}}}{\sqrt{\mathbf{q}_{\mathbf{0}, \mathbf{i}}^{\mathbf{T}} \mathbf{M} \mathbf{q}_{\mathbf{0}, \mathbf{i}}}}, \quad(i=1,2, \ldots, 6) .
$$

An arbitrary solution of the eigenvector $\mathbf{q}$ can be produced with the linear combination of the eigenvectors:

$$
\mathbf{q}(t)=\sum_{\mathbf{i}=\mathbf{1}}^{6} \mathbf{q}_{\mathbf{n}, \mathbf{i}} a_{i}(t) .
$$

Substituting (17) into (13), multiplying the term with $\mathbf{q}_{\mathbf{n}, \mathbf{i}}^{\mathbf{T}}$, and assume that a real system has dissipation, thus Lehr's damping value $(\xi)$ is taken into consideration, we can get the following form of the differential equation:

$$
\ddot{a}_{i}+2 \xi_{i} \alpha_{i} \dot{a}_{i}+\alpha_{i}^{2} a_{i}=Q_{a, i}, \quad(i=1,2, \ldots, 6),
$$

where $Q_{a, i}=\mathbf{q}_{\mathbf{n}, \mathbf{i}}^{\mathbf{T}} \mathbf{Q}_{\mathbf{F}}$. The ordinary differential equation can be solved analytically and also numerically.

\subsection{Simulation results}

The screening machine is excited by three interconnected eccentric mass as shown in Figure 4.

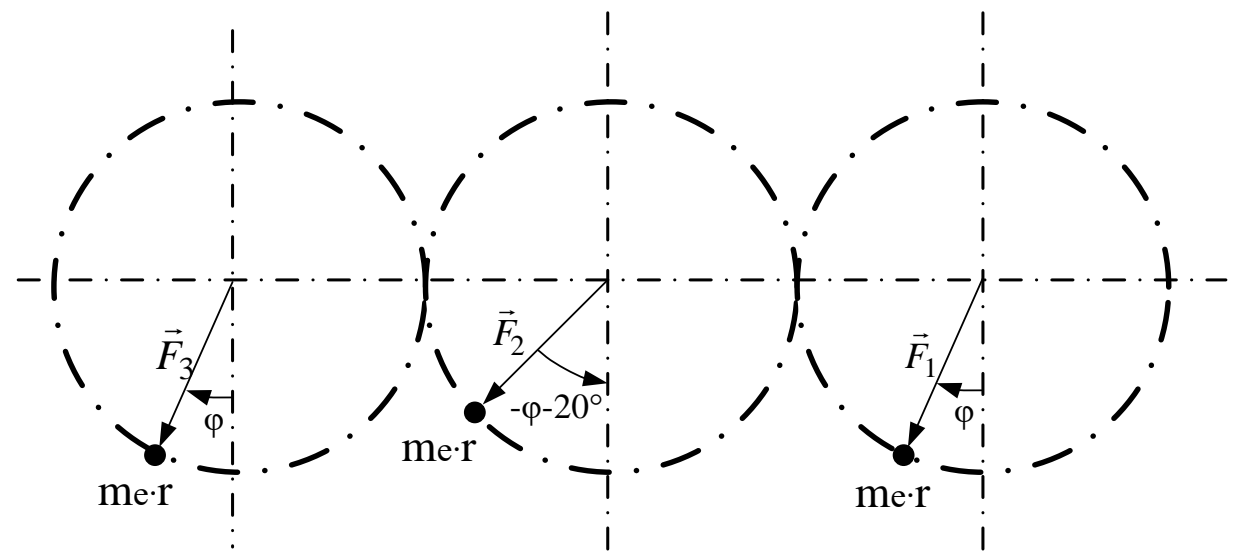

Figure 4. The phases of the three eccentric masses

Pre-measurements were performed in order to determine the characteristic frequency of the working system. The dominate frequency was $13.2 \mathrm{~Hz}$ due to the rotation of the eccentric masses. The calculated eccentricity $m_{e} r=1.89 \mathrm{kgm}$.

We assume that the points of application of the forces due to eccentricity $\vec{r}_{F, 1}=(-0.125 \cdot \vec{\imath}+0.75 \cdot \vec{\jmath}-$ $0.5 \cdot \vec{k}), \vec{r}_{F, 2}=(-0.125 \cdot \vec{\imath}+0.25 \cdot \vec{\jmath}-0.5 \cdot \vec{k})$, and $\vec{r}_{F, 3}=(-0.125 \cdot \vec{\imath}-0.25 \cdot \vec{\jmath}-0.5 \cdot \vec{k})$.

The exciting forces due to eccentricity at steady-state working method: $\vec{F}_{1}=\vec{F}_{3}=13000 \cdot \cos (\omega \cdot t) \vec{\jmath}+$ $13000 \cdot \sin (\omega \cdot t) \vec{k}, \quad \vec{F}_{2}=13000 \cdot \cos \left(-\omega \cdot t-20^{\circ}\right) \vec{\jmath}+13000 \cdot \sin \left(-\omega \cdot t-20^{\circ}\right) \vec{k}, \quad$ where $\omega=$ $82.94 \frac{\mathrm{rad}}{\mathrm{s}}$. The angular velocity has a ramp up time (see Figure 5). 


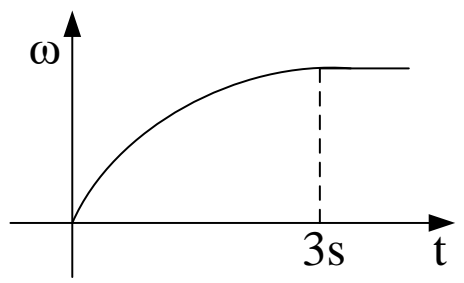

Figure 5. The ramp up time of the angular velocity

$$
\begin{gathered}
\text { if } t<3 \quad \omega=\left(-t^{2}-12 t\right) / 36 \\
\text { if } t \geq 3 \quad \omega=82.94 \mathrm{rad} / \mathrm{s} .
\end{gathered}
$$

In the course of transient response the angular velocity of the eccentric masses are changing according to (19). Therefore numerical method is performed to determine the vibration of the system.

Natural frequencies determined by the modal analysis: $f_{1}=2.5 \mathrm{~Hz}, f_{2}=2.81 \mathrm{~Hz}, f_{3}=3.06 \mathrm{~Hz}, f_{4}=$ $5.3 \mathrm{~Hz}, f_{5}=5.43 \mathrm{~Hz}$, and $f_{6}=5.58 \mathrm{~Hz}$. These frequencies are bigger than the measured operating frequency of the system.

The motions in $y$ direction of the following two points $\vec{r}_{P, 1}=-3.364 / 2 \cdot \vec{\imath}-5.604 / 2 \cdot \vec{\jmath}-0.5 \cdot \vec{k}$, and $\vec{r}_{P, 2}=3.364 / 2 \cdot \vec{\imath}-5.604 / 2 \cdot \vec{\jmath}-0.5 \cdot \vec{k}$ are determined. The displacements versus time in $y$ directions are shown in Figure 6 and Figure 7.

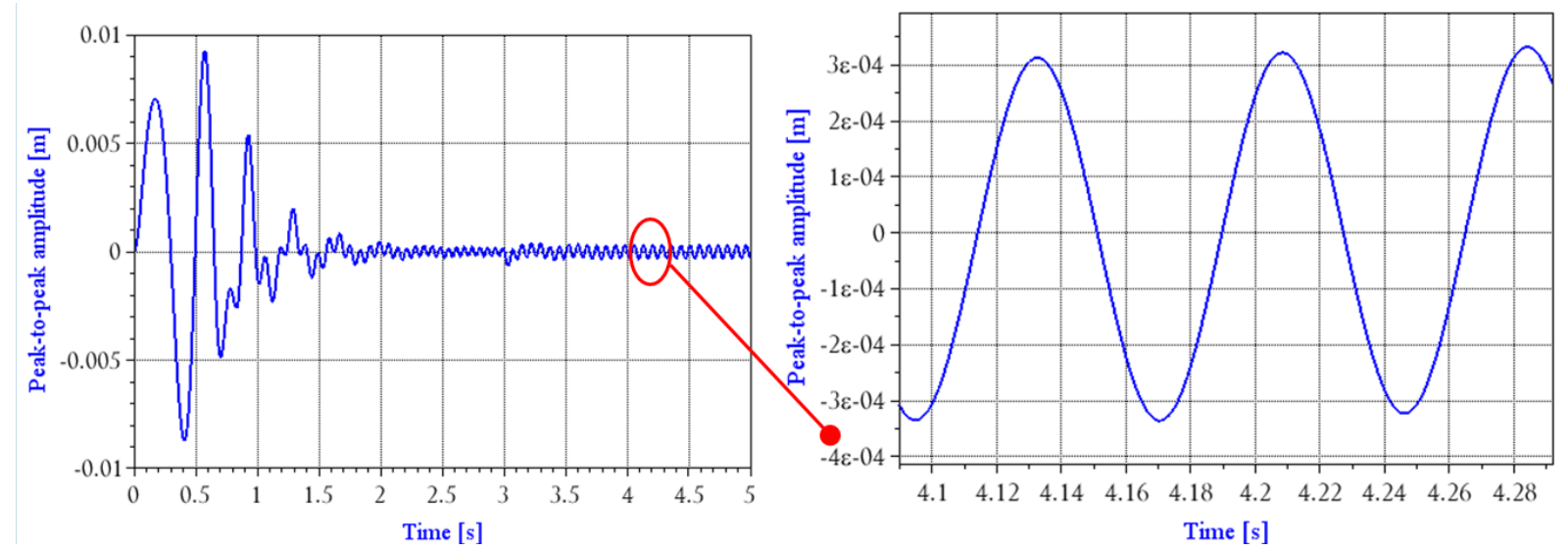

Figure 6. The simulation result of the point 1

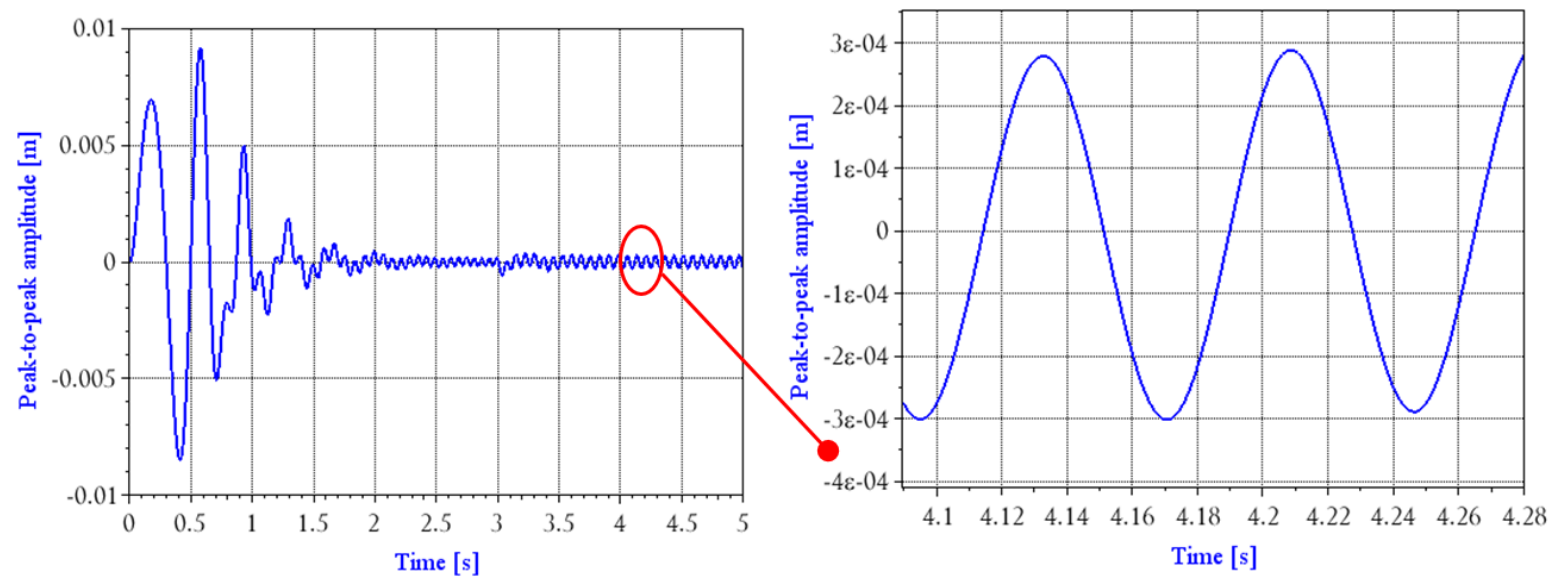

Figure 7. The simulation result of the point 2 


\subsection{Measurements with a laser triangulation unit}

Vibration measurements have been performed in order to detect the peak-to-peak amplitude of the points $\vec{r}_{P, 1}$ and $\vec{r}_{P, 2}$ on the screening machine in the course of steady state operation. Micro-epsilon ILD2220-200 laser triangulation unit [10] was used to perform measurements. During the measurements, the water pump was switched off and no gravel was applied. The result of a test measurement of the point 1 is shown in Figure 8.

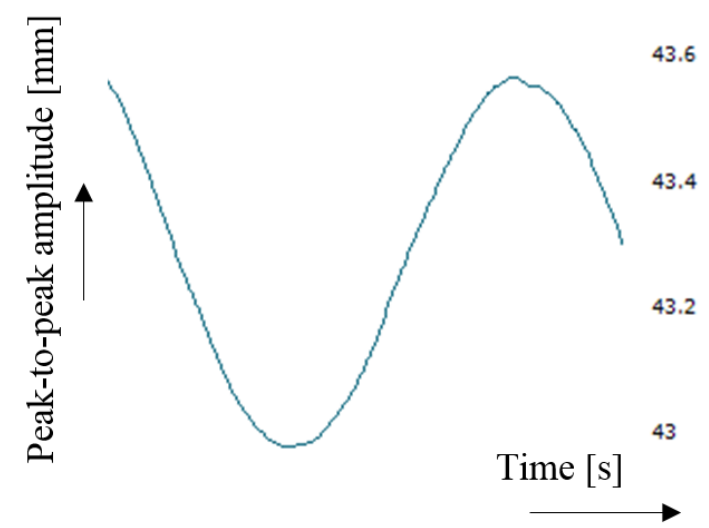

Figure 8. Laser measurement of the point 1

The peak-to-peak amplitude of point $\vec{r}_{P, 1}$ is approximately $0.66 \mathrm{~mm}$, and the dominant frequency is 13.2 Hz. The results of the point 2 can be seen in Figure 9.

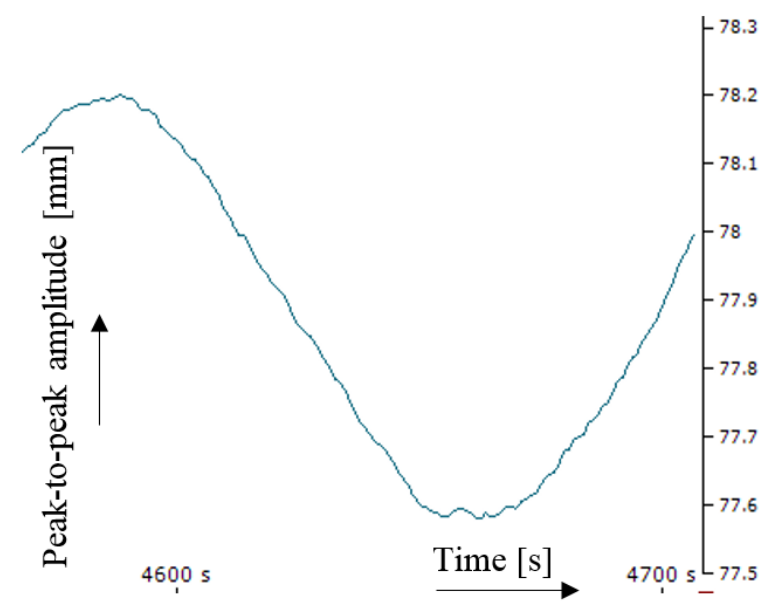

Figure 9. The result of the point 2

The peak-to-peak amplitude of the point $\vec{r}_{P, 2}$ is about $0.6 \mathrm{~mm}$.

The simulation results show a good correlation with the test measurements. The dynamical model of the system can be used to predict in a predefined point the coordinate displacement values to the transient starting condition or the steady-state status.

\section{Conclusions}

This paper was dealt with the modelling and simulation of the horizontal screening machine. The machine was considered as a rigid cuboid. Linear vibration approach has been used to obtain the natural 
frequencies, and the peak-to-peak amplitudes of the transient response and the steady-state status of the model of the vibration screen.

The numerical simulation and the measurements were performed in two points. The applicability of the model has been verified by the measurements. The frequencies and the displacements in $y$ direction have been determined by the use of laser triangulation technique. The result of the measurements and the simulations showed a good correlation.

\section{Acknowledgement}

The described article was carried out as part of the EFOP-3.6.1-16-2016-00011 "Younger and Renewing University - Innovative Knowledge City - institutional development of the University of Miskolc aiming at intelligent specialisation" project implemented in the framework of the Szechenyi 2020 program. The realization of this project is supported by the European Union, co-financed by the European Social Fund.

\section{References}

[1] Makinde, O. A., Ramatsetse, B. I., Mpofu, K. (2015) 'Review of vibrating screen development trends: Linking the past and the future in mining machinery industries', International Journal of Mineral Processing, 145, pp. 17-22.

[2] Ramatsetse, B., Mpofu, K., Makinde, O., Campbell, H. M., Engelbrecht, C. (2016) 'Design and structure optimization of a reconfigurable vibrating screen for the mining and mineral processing industries', New technology and innovation in the Minerals Industry Colloquium, Southern African Institute of Mining and Metallurgy, pp. 125-144.

[3] Zhao, Y. M., Liu, C. S., He, X. M., Cheng-yong, Z., Yi-bin, W., Zi-ting, R. (2009) 'Dynamic design theory and application of large vibrating screen', Procedia Earth \& Planetary Science, 1(1), pp. 776-784.

[4] Diamond Equipment Inc. 'TRIO Horizontal Screens - High Effective Screening Solutions ᄀ', catalogue, United States, pp. 1-8.

[5] Weir Group PLC (2020) 'Trio® TTH series screen specifications', Glasgow, https://www.global.weir/products/product-catalogue/trio-tth-series-screens/

[6] Bathe, K. J. (1996) 'Finite Element Procedures', Prentice Hall Inc., Upper Saddle River, New Jersey

[7] Wang, J., Ricardo, B. (2019) 'Squashing Method for Moment of Inertia Calculations', The Physics Teacher, 57, pp. 155-158.

[8] Sankyo Oilless Industry Inc. 'General Description of Rubber Spring', datasheet, pp. 501-512.

[9] Szabó, T., (2011) 'Dynamics of Structures', Universitas-Győr Nonprofit Kft., Győr (in Hungarian)

[10] MICRO-EPSILON Headquarters 'Laser Triangulation Displacement Sensors', datasheet, Y9761188-D091120DGO, Ortenburg, Germany, pp. 1-36. 